\title{
Study on Robust Fault Detection For Linear Discrete Time-Varying Systems With Measurement Missing Chun-peng Wang ${ }^{1, a, *}$, Jian Guo ${ }^{2, b}$

\author{
${ }^{1}$ School of Electrical Engineering, Binzhou Polytechnic, Binzhou 256603, China \\ afuwa08@163.com, b232463158@qq.com
} \\ ${ }^{2}$ School of Mechanical Engineering, Binzhou Polytechnic, Binzhou 256603, China
}

\begin{abstract}
Keywords: Robust fault detection filter, Missing measurements, $H_{\infty}$ filtering, Linear discrete time-varying systems, Linear matrix inequality.
\end{abstract}

\begin{abstract}
In this study, the robust fault detection problem is investigated for a class of linear discrete time-varying systems with multiple measurement packet dropouts. The missing measurements are modeled as a linear function of the stochastic variable satisfying Bernounlli random binary distribution. A robust fault detection filter based on $H_{\infty}$ filtering is designed to make residual error system be exponentially mean-square stable and satisfy a prescribed disturbance attenuation level. A sufficient condition is derived in terms of linear matrix inequalities. Numerical examples are given to illustrate the effectiveness of the proposed method. The simulation result has showed the effectiveness and applicability of the obtained approach.
\end{abstract}

\section{Introduction}

In recent years, the robust fault detection method based on $H_{\infty}$ filtering ${ }^{[1-7]}$ has attracted much attention of researchers and has gained rather rich research results. However, most of these researches are aimed ate the linear constant systems, and the researches on linear time-varying systems are relatively few. The problem of robust fault detection and isolation for a class of discrete time linear parameter varying systems with time-varying delay has been studied in [4]. The problem of $H_{\infty}$ fault estimation for a class of linear discrete time-varying systems with $l_{2}$-norm -bounded disturbance has been discussed in [5].

At present, with the general application of network in industrial production, the phenomenon of time-delay and missing measurements has been widespread in the process of data transmission. In the case of incomplete measurement information, the problem of fault detection has become the focus of current research. In [6] and [7], the problem of the filtering and fault detection with random time-delays and measurement packet dropouts has been discussed respectively. The design of fault detection filter for linear discrete-time systems with random time-delays and measurement packet dropouts has been studied in [8]. In [9] and [10], the fault detection of a class of linear time-varying systems with multiple packet dropouts and multiple missing measurements has been studied respectively. But the above literatures are not aimed at linear discrete time-varying systems except for the [9] and [10].

In this paper, the robust fault detection problem is investigated for a class of linear discrete time-varying systems with multiple measurement packet dropouts. The missing measurements are described by a linear function of the stochastic variable satisfying Bernounlli random binary distribution. By using $H_{\infty}$ control theory, the robust fault detection filter (RFDF) design can be formulated as an $H_{\infty}$ filtering problem. A sufficient condition for the existence of the desired fault detection filter is established in terms of linear matrix inequalities (LMI), and a numerical example is provided to illustrate the effectiveness and applicability of the proposed design method. 


\section{Problem formulation}

Consider the following class of linear discrete time-varying systems:

$$
\left\{\begin{array}{l}
x(k+1)=A(k) x(k)+B_{d}(k) d(k)+B_{f}(k) f(k) \\
y(k)=C(k) x(k)+D_{d}(k) d(k)+D_{f}(k) f(k)
\end{array}\right.
$$

where $x(k) \in R^{n}$ is the state vector; $y(k) \in R^{q}$ is the signal to be estimated; $d(k) \in R^{p}$ is the unknown disturbance input; $f(k) \in R^{r}$ is the fault to be detected; $A(k), B_{d}(k), B_{f}(k), C(k)$, $D_{d}(k)$ and $D_{f}(k)$ are known real constant matrices with appropriate dimensions.

The insertion of network cable between measurement node and fault detection filter causes measurement missing phenomena. Furthermore, the measurement can be described by

$$
\psi(k)=\delta(k) y(k)+(1-\delta(k)) \psi(k-1)
$$

where $\psi(k) \in R^{q}$ is the measured output, and the stochastic variable $\delta(k) \in R$ is a Bernoulli distributed white sequence with

$$
\left\{\begin{array}{l}
\operatorname{Prob}\{\delta(k)=1\}=E\{\delta(k)\}=\delta \\
\operatorname{Prob}\{\delta(k)=0\}=1-E\{\delta(k)\}=1-\delta
\end{array}\right.
$$

and $\delta \in R \delta \in(0,1]$ is a known positive scalar.

The core module of the fault detection based on $H_{\infty}$ filtering is the generation of residuals. In the case of system Eq. 1 is said to be asymptotically stable, consider the following RFDF for system with multiple measurement packet dropouts:

$$
\left\{\begin{array}{l}
\hat{x}(k+1)=A_{F}(k) \hat{x}(k)+B_{F}(k) \psi(k) \\
r(k)=C_{F}(k) \hat{x}(k)
\end{array}\right.
$$

where $\hat{x}(k) \in R^{n}$ is the state estimate, $r(k) \in R^{r}$ is the so-called residual that is compatible with the fault vector, and $A_{F}(k) \in R^{n \times n}, B_{F}(k) \in R^{n \times p}$ and $C_{F}(k) \in R^{p \times n}$ are filter parameters to be determined.

Define the augmented state vector $\xi(k)=\left[\begin{array}{lll}x^{T}(k) & \hat{x}^{T}(k)\end{array}\right]^{T}$, Denote $\omega(k)=\left[\begin{array}{ll}d^{T}(k) & f^{T}(k)\end{array}\right]^{T}$, $r_{e}(k)=r(k)-f(k)$, the augmented system formed by system Eq. 1, Eq. 2 and the filter Eq. 4 can be expressed by

$$
\left\{\begin{array}{l}
\xi(k+1)=A_{\xi 1}(k) \xi(k)+A_{\xi 2}(k) \xi(k-1)+B_{\xi}(k) \omega(k) \\
r_{e}(k)=C_{1}(k) \xi(k)+D_{1}(k) \omega(k)
\end{array}\right.
$$

where

$$
\begin{aligned}
& A_{\xi 1}(k)=A_{1}(k)+\delta(k) A_{2}(k), \quad A_{1}(k)=\left[\begin{array}{cc}
A(k) & 0 \\
0 & A_{F}(k)+I
\end{array}\right], \quad A_{2}(k)=\left[\begin{array}{cc}
0 & 0 \\
B_{F}(k) C(k) & -I
\end{array}\right], \\
& A_{\xi 2}(k)=A_{3}(k)+\delta(k) A_{4}(k), \quad A_{3}(k)=\left[\begin{array}{cc}
0 & 0 \\
0 & -A_{F}(k)
\end{array}\right], A_{4}(k)=\left[\begin{array}{cc}
0 & 0 \\
0 & A_{F}(k)
\end{array}\right], \\
& B_{\xi}(k)=B_{1}(k)+\delta(k) B_{2}(k), \quad B_{1}(k)=\left[\begin{array}{cc}
B_{d}(k) & B_{f}(k) \\
0 & 0
\end{array}\right], \quad B_{2}(k)=\left[\begin{array}{cc}
0 \\
B_{F}(k) D_{d}(k) & B_{F}(k) D_{f}(k)
\end{array}\right],
\end{aligned}
$$


$C_{1}(k)=\left[\begin{array}{ll}0 & C_{F}(k)\end{array}\right], \quad D_{1}(k)=\left[\begin{array}{ll}0 & -I\end{array}\right]$.

The design problem stated above will be referred to as the robust filtering problem with networked time-delay and missing measurements. More specifically, we aim to design the filter Eq. 4 such that the following requirements are satisfied simultaneously: for a given $\gamma>0$, the system Eq. 5 is exponentially mean-square stable and

$$
E\left\|r_{e}(k)\right\|_{2}<\gamma E\|\omega(k)\|_{2}
$$

where $\gamma>0$ is a prescribed scalar.

The finial important task of fault detections is the residual evaluation. Based on the designed fault detection system, the residual evaluation function $J(k)$ and the threshold $J_{t h}{ }^{[3]}$ are determined as follows:

$$
\left\{\begin{array}{c}
J(k)=\left\{\sum_{k=k_{0}}^{k_{0}+N} r^{T}(k) r(k)\right\}^{1 / 2} \\
J_{t h}=\sup _{d(k) \in l_{2}, f(k)=0} E\{J(k)\}
\end{array}\right.
$$

where $k_{0}$ denotes the initial evaluation time instant, $N$ denotes the length of time window.

Based on the above, the fault can be detected by using the following logical relationship:

$$
\left\{\begin{array}{c}
J(k)>J_{t h} \Rightarrow \text { abnormal } \Rightarrow \text { fault } \\
J(k) \leq J_{t h} \Rightarrow \text { normal }
\end{array}\right.
$$

\section{Design of robust fault detection filter}

Theorem 1: For a given scalar $\gamma>0$, the system Eq. 5 is exponentially mean-square stable and achieves the $H_{\infty}$-norm constraint Eq. 6 for all nonzero $\omega(k)$, if there exist positive definite matrices $P(k)$ and $Q$ satisfying equation Eq. 9.

$$
\left[\begin{array}{cccccc}
-P(k)+Q & * & * & * & * & * \\
0 & -Q & * & * & * & * \\
0 & 0 & -\gamma^{2} I & * & * & * \\
\Xi_{41} & \Xi_{42} & \Xi_{43} & -(1-\delta) P(k+1) & * & * \\
\Xi_{51} & \Xi_{52} & \Xi_{53} & 0 & -\delta P(k+1) & * \\
C_{1}(k) & 0 & D_{1}(k) & 0 & 0 & -I
\end{array}\right]<0
$$

where $\Xi_{41}=(1-\delta) P A_{1}(k), \quad \Xi_{42}=(1-\delta) P(k+1) A_{3}(k), \quad \Xi_{43}=(1-\delta) P(k+1) B_{1}(k)$, $\Xi_{51}=\delta P(k+1)\left(A_{1}(k)+A_{2}(k)\right), \quad \Xi_{52}=\delta P(k+1)\left(A_{3}(k)+A_{4}(k)\right), \quad \Xi_{53}=\delta P(k+1)\left(B_{1}(k)+B_{2}(k)\right)$.

Remark 1: The proof of Theorem 1 would require to be combined with definition in [12] and lemma 1 in [13]. The specific proof process would be omitted.

Corollary 1: For a given scalar $\gamma>0$, the system Eq. 5 is exponentially mean-square stable and achieves the $H_{\infty}$-norm constraint Eq. 6 for all nonzero $\omega(k)$, if there exist positive definite matrices $W(k), Y(k), Z_{1}(k)$ and $Z_{3}(k)$, matrices $Z_{2}(k), M(k), N(k)$ and $S(k)$ satisfying Eq. 10. 


$$
\left[\begin{array}{cccccc}
\psi_{11} & * & * & * & * & * \\
0 & \psi_{22} & * & * & * & * \\
0 & 0 & -\gamma^{2} I & * & * & * \\
\psi_{41} & \psi_{42} & \psi_{43} & \psi_{44} & * & * \\
\psi_{51} & 0 & \psi_{53} & 0 & \psi_{55} & * \\
\psi_{61} & 0 & \psi_{63} & 0 & 0 & -I
\end{array}\right]<0
$$

where $\psi_{11}=\left[\begin{array}{cc}-X(k)+Z_{1}(k) & * \\ -X(k)+Z_{2}(k) & -Y(k)+Z_{3}(k)\end{array}\right], \quad \psi_{22}=\left[\begin{array}{cc}-Z_{1}(k) & * \\ -Z_{2}(k) & -Z_{3}(k)\end{array}\right]$,

$\psi_{41}=\left[\begin{array}{cc}(1-\delta) X(k+1) A(k) & (1-\delta) X(k+1) A(k) \\ (1-\delta)(Y(k+1) A(k)+M(k)+X(k)-Y(k)) & (1-\delta) Y(k+1) A(k)\end{array}\right]$,

$\psi_{42}=\left[\begin{array}{cc}0 & 0 \\ -(1-\delta) M(k) & 0\end{array}\right], \quad \psi_{43}=\left[\begin{array}{cc}(1-\delta) X(k+1) B_{d}(k) & (1-\delta) X(k+1) B_{f}(k) \\ (1-\delta) Y(k+1) B_{d}(k) & (1-\delta) Y(k+1) B_{f}(k)\end{array}\right]$,

$\psi_{44}=\left[\begin{array}{cc}-(1-\delta) X(k+1) & * \\ -(1-\delta) X(k+1) & -(1-\delta) Y(k+1)\end{array}\right]$,

$\psi_{51}=\left[\begin{array}{cc}\delta X(k+1) A(k) & \delta X(k+1) A(k) \\ \delta(Y(k+1) A(k)+S(k) C(k)+M(k)) & \delta(Y(k+1) A(k)+S(k) C(k))\end{array}\right]$,

$\psi_{53}=\left[\begin{array}{cc}\delta X(k+1) B_{d}(k) & \delta X(k+1) B_{f}(k) \\ \delta\left(Y(k+1) B_{d}(k)+S(k) D_{d}(k)\right) & \delta\left(Y(k+1) B_{f}(k)+S(k) D_{f}(k)\right)\end{array}\right]$,

$\psi_{55}=\left[\begin{array}{cc}-\delta X(k+1) & * \\ -\delta X(k+1) & -\delta Y(k+1)\end{array}\right], \psi_{61}=\left[\begin{array}{cc}N(k) & 0\end{array}\right], \psi_{63}=\left[\begin{array}{cc}0 & -I\end{array}\right]$.

Proof : Partitioning matrices $P(k)$ and $U(k)=P^{-1}(k)$ into the following block forms:

$$
P(k)=\left[\begin{array}{cc}
P_{1}(k) & P_{2}(k) \\
P_{2}^{T}(k) & P_{3}(k)
\end{array}\right], U(k)=P^{-1}(k)=\left[\begin{array}{cc}
U_{1}(k) & U_{2}(k) \\
U_{2}^{T}(k) & U_{3}(k)
\end{array}\right],
$$

where $P_{1}(k) \in R^{n \times n}$ and $U_{1}(k) \in R^{n \times n}$ are symmetric positive definite matrices, $P_{2}(k)$ and $U_{2}(k)$ are two non-singular matrices.

Define the invertible matrices as follows:

$$
J_{1}(k)=\left[\begin{array}{cc}
U_{1}(k) & I \\
U_{2}^{T}(k) & 0
\end{array}\right], \quad J_{2}(k)=\left[\begin{array}{cc}
I & P_{1}(k) \\
0 & P_{2}^{T}(k)
\end{array}\right],
$$

which implies that $P(k) J_{1}(k)=J_{2}(k)$, and let $J_{1}^{T}(k) Q J_{1}(k)=\left[\begin{array}{cc}U_{1}(k) Z_{1}(k) U_{1}(k) & U_{1}(k) Z_{2}^{T}(k) \\ Z_{2}(k) U_{1}(k) & Z_{3}(k)\end{array}\right]$.

Performing congruence transformation to Eq. 9 by the transformational matrix $\Xi_{1}(k)$, then preand post-multiplying the resulting LMI of the above transformation by the transformational matrix $\Xi_{2}(k)$ and defining the following matrix variables: $U_{1}^{-1}(k)=W(k), P_{1}(k)=Y(k)$, $P_{2}(k+1) A_{F}(k) U_{2}^{T}(k)=M(k) W^{-1}(k), C_{F}(k) U_{2}^{T}(k)=N(k) W^{-1}(k), \quad P_{2}(k+1) B_{F}(k)=S(k)$, we can arrive at the result $(10)$ in Corollary 1 , where

$\Xi_{1}(k)=\operatorname{diag}\left\{J_{1}(k), \quad J_{1}(k), \quad I \quad J_{1}(k+1), \quad J_{1}(k+1), \quad I\right\}$, $\Xi_{2}(k)=\operatorname{diag}\left\{W^{-1}(k), \quad I, W^{-1}(k), \quad I, \quad I, \quad I, W^{-1}(k+1), \quad I, W^{-1}(k+1), \quad I, \quad I\right\}$.

This ends the proof.

Moreover, the desired filter parameters of Eq. 4 can be given by 


$$
A_{F}(k)=(X(k)-Y(k))^{-1} M(k), B_{F}(k)=(X(k)-Y(k))^{-1} S(k), C_{F}(k)=N(k)
$$

Remark 2: Let $g=\gamma^{2}$. The sub-optimal robust filtering problem for discrete-time uncertain systems with one-step communication delay and measurement missing can be represented as follows:

$$
\min _{W(k), Y(k), Z_{1}(k), Z_{2}(k), Z_{3}(k), M(k), N(k), S_{1}(k)} g
$$

The parameters of the sub-optimal filters can be determined by Eq. 12, and the sub-optimal $H_{\infty}$ attenuation levels are given by $\gamma_{\text {opt }}=\sqrt{g^{*}}$, where $g^{*}$ are the sub-optimal solutions of the corresponding convex optimization problems.

\section{Simulation example}

In this section, a numerical example is proved to illustrate the effectiveness and applicability of the proposed method. Consider the system described by (1) with parameters as follows:

$$
\begin{gathered}
A=\left[\begin{array}{cc}
0.1 e^{-k / 100} & 0.9^{k} \\
0.85 & -0.3 \sin (k)
\end{array}\right], B_{d}=\left[\begin{array}{l}
0.5 \\
0.2
\end{array}\right], \quad B_{f}=\left[\begin{array}{c}
0.3 \sin (k) \\
0.4
\end{array}\right], \\
C=\left[\begin{array}{ll}
0.2 & 0.3
\end{array}\right], D_{d}=0.3, \quad D_{f}=0.8 .
\end{gathered}
$$

For $k=0,1, \ldots, 200$, the unknown input $d(k)$ is supposed to be random noise which is uniformly distributed over $[-0.5,0.5]$, and the fault signal $f(k)$ is given as:

$$
f(k)=\left\{\begin{array}{cc}
1, & k=60, \mathrm{~L}, 140 \\
0, & \text { else }
\end{array}\right.
$$

By using equation (7), the threshold $J_{t h}=0.0039428$ can be obtained. The simulation results indicate that the evaluation function $J(k)=0.0045429>J_{t h}$, so the appeared fault can be detected after 6 time steps. Fig. 1 shows the generated residual signal $r(k)$ without stepwise fault signal. Fig.2 demonstrates the evolution of residual evaluation function, in which the blue dashed line stands for fault-free case and the red solid line for the fault case. The simulation results are given in Fig. 1 and Fig.2, from which effectiveness of the method is illustrated.

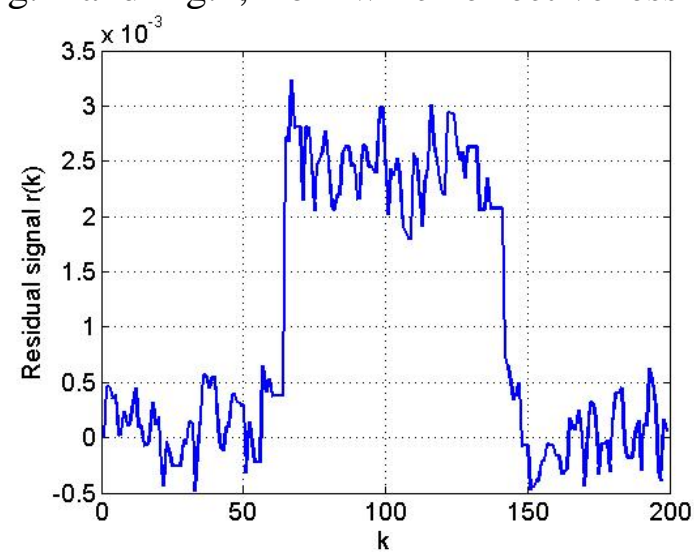

Fig.1 Residual $r(k)$ with Stepwise fault signal

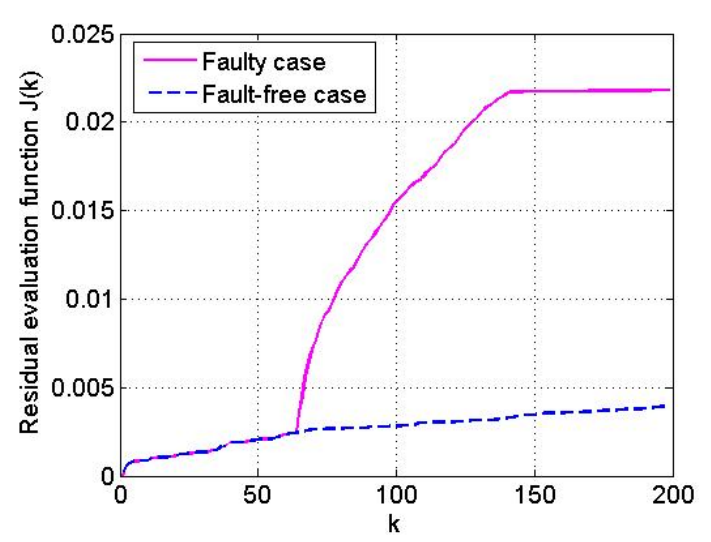

Fig.2 Evolution of residual evaluation $J(k)$

\section{Conclusions}

The problem of RFDF has been considered in this paper for a class of linear discrete 
time-varying systems with multiple measurement packet dropouts. The RFDF based on $H_{\infty}$ filtering has been used as residual generator. Then the design problem of $R F D F$ has been reduced to $H_{\infty}$ filtering problem. A sufficient condition for the existence of the RFDF has been established by means of LMI, and the parameters of the RFDF are determined directly by solutions of the LMI. The simulation result has showed the effectiveness and applicability of the obtained approach.

\section{References}

[1] Xu S X, Chen T W, Lam J. Robust $H_{\infty}$ filtering for uncertain Markovian jump systems with mode-dependent time delays[J]. IEEE Trans. Automatic Control, 2003, 48 (5):900-907.

[2] Wang Z D, Daniel W C, Liu X H. Robust filtering under randomly varying sensor delay with variance constraints [J]. IEEE Trans. On Circuits \& Systems, 2004, 51(6):320-326.

[3] Zhong Maiying, Ding Steven X, Lam James, el at. An LMI approach to design robust fault detection filter for uncertain LTI systems [J].Automatic, 2003, 39(3):543-550.

[4] Wang Hongru, Wang Changhong, Gao huijun. Robust fault detection and isolation for a class of discrete time linear parameter varying systems with time delays [J].Journal of Harbin Institute of Technology, 2007:39(9):1350-1355.

[5] Liu Dianrui, Zhao Huihong, Zhong Maiying. The $H_{\infty}$ fault estimation for linear discrete time-varying systems[J].Journal of Shandong University (Engineering Science), 2008: 38(4):11-16.

[6] Wang Wu,Yang Fuwen. $H_{\infty}$ filter design for discrete-time systems with missing measurements[J]. Acta Automatica Sinica, 2006, 32(1):107-111.

[7] Ruan Yu-bin, Wang Wu, Yang Fu-wen. Fault detection filter for networked systems with missing measurements [J]. Control Theory and Applications, 2009,26(3):291-295.

[8] He Xiao, Wang Zi dong, and Zhou Dong hua. Networked fault detection with random communication delays and packet losses. International journal of systems Science,2008, 39(11):1045-1054.

[9] Li Yue-yang, Zhong Mai-ying. On designing robust $H_{\infty}$ fault detection filter for linear discrete time-varying systems with multiple packet dropouts [J]. Acta Automatica Sinica, 2010, 36(12): 1788- 1796

[10] Li Yue-yang, Zhong Mai-ying. Fault detection filter design for linear discrete time-varying systems with multiple missing measurements [J]. Control Engineering of China, 2011, 18(4):640-644

[11] Wang Zidong, Yang Fuwen, Ho D C, et al. Robust $H_{\infty}$ filtering for stochastic time-delay systems with measurements [J].IEEE Transactions on signal processing, 2006, 54(7):2579-2587.

[12] Yang Fuwen, Wang Zidong, Hung Y S et al. $H_{\infty}$ Control for networked systems with random communication delays [J].IEEE Trans .on Automatic Control, 2006, 51(3):511-518 